\title{
What Web-Based Intervention for Chronic Cancer- Related Fatigue Works Best for Whom? Explorative Moderation Analyses of a Randomized Controlled Trial.
}

Melanie P. J. Schellekens ( $\square$ mschellekens@hdi.nl )

Helen Dowling Instituut https://orcid.org/0000-0001-8397-7674

Fieke Z Bruggeman-Everts

Helen Dowling Institute: Helen Dowling Instituut

Marije Wolvers

Helen Dowling Institute: Helen Dowling Instituut

Miriam M.R. Vollenbroek-Hutten

Universiteit Twente

Marije L. van der Lee

Tilburg University

\section{Research Article}

Keywords: Chronic Cancer-Related Fatigue, Fatigue, Cancer survivors, Mindfulness-Based Cognitive Therapy, activity intervention, moderation analysis

Posted Date: February 1st, 2022

DOI: https://doi.org/10.21203/rs.3.rs-1166105/v1

License: (c) (i) This work is licensed under a Creative Commons Attribution 4.0 International License.

Read Full License 


\section{Abstract}

Purpose: Approximately $25 \%$ of cancer patients suffers from chronic cancer-related fatigue (CCRF), which is a complex, multifactorial condition. While there are evidence-based interventions, it remains unclear what treatment works best for the individual patient. This study explored whether baseline characteristics moderated the effect of web-based Mindfulness-Based Cognitive Therapy (eMBCT) versus Ambulant Activity Feedback (AAF) and a psycho-education control group (PE) on fatigue in patients suffering from CCRF.

Methods: In a randomized controlled trial, participant suffering from CCRF participated in either eMBCT, AAF or PE. Complete data of the treatment-adherent sample ( $\geq 6$ sessions) was used to explore whether sociodemographic, clinical and psychological characteristics at baseline moderated the intervention effect on fatigue severity at 6 months.

Results: A trend suggested that baseline fatigue severity and fatigue catastrophizing moderated the intervention effect. That is, at low levels of fatigue severity and catastrophizing, patients benefited more from AAF than from eMBCT and at high levels of fatigue severity and catastrophizing, patients benefited more from $\mathrm{EMBCT}$ than from $\mathrm{PE}$.

Conclusions: This study found some preliminary evidence on what treatment works best for the individual suffering from CCRF. These findings suggest a potential gain in effectiveness of personalizing treatment. An alternative and more person-centered approach that might help us further in answering the question 'what treatment works best for whom?' is discussed.

\section{Introduction}

Fatigue is one of the most prevalent and disrupting side effects of cancer and its treatment. This fatigue differs from typical tiredness as it is not alleviated by rest or sleep, nor is it proportional to recent exertion [1]. In approximately $25 \%$ of patients, it persists for months to years after completion of cancer treatment [1]. This persisting fatigue is defined as chronic cancer-related fatigue (CCRF). CCRF interferes with patients' ability to work, their daily life activities and social relationships and is often accompanied by distress $[1,2]$.

Evidence suggests CCRF is a complex multifactorial condition, affected by a range of physiological (e.g. inflammation), clinical (e.g. comorbidities, cancer treatment), and psychosocial factors (e.g. distress) [1]. Following the multifactorial etiology of CCRF, several types of treatments have proven effective in helping patients cope with CCRF. These include both psychosocial interventions as well as physical activity interventions [3]. So far, it remains unknown what intervention is most suitable for which patient. In order to provide patients with the best and most efficient care we need to answer the question 'What works best for whom?' Therefore, characteristics that influence the direction or magnitude of the effect of such interventions on cancer-related fatigue need to be identified. 
Recently, a three-armed randomized controlled trial compared the effects of two online interventions (a psychologist-guided web-based MBCT (eMBCT) and a physiotherapist-guided ambulant activity feedback (AAF)) to an unguided active control group receiving psycho-education (PE) in severely fatigued cancer patients [4]. Results showed that both $\mathrm{EMBCT}$ and $\mathrm{AAF}$ were superior to $\mathrm{PE}$ in reducing fatigue [4]. This three-armed design provides an unprecedented opportunity to conduct moderation analyses and study for which subgroup of patients what type of intervention (i.e. eMBCT, AAF or PE) is most beneficial. The aim of the current study is to explore the moderator effects of sociodemographic, clinical and psychological characteristics on the effect of eMBCT, AAF and PE on fatigue in patients suffering from CCRF.

\section{Methods}

This moderation study was embedded in a three-armed randomized controlled trial [5]. The study was approved by the Twente Medical Ethical Committee (P12-26).

\section{Participants and procedure}

Cancer survivors were recruited via various channels (e.g. patient organizations, social media, newspapers, health care professionals). Patients were invited to follow a web-based intervention for their fatigue but did not receive the exact content of the interventions in the advertisements. Inclusion criteria were: having finished curative-intent cancer treatment at least three months previously after any type of cancer diagnosis, suffering from severe fatigue ever since ( $\geq 35$ on the Checklist Individual Strength Fatigue Severity [CIS-FS] subscale [4]), being $\geq 18$ years old at disease onset, no current or former severe psychiatric comorbidity (e.g. suicidal ideation, psychosis), no current substance abuse, no cancer recurrence during study participation, and no dependence on wheelchair for daily activities.

After providing written informed consent, eligible patients were randomised to eMBCT, AAF or PE [4]. Participants filled out questionnaires prior randomization (baseline, T0), one week after the 9-week intervention period (T1) and at follow-up (6 months, T2).

\section{Interventions}

\section{eMBCT}

The eMBCT-program is a 9-week web-based psychologist-guided intervention, based on the original MBCT protocol [6] and tailored to patients suffering from CCRF by including cancer and fatigue-related psycho-education, and adapted movement exercises [7]. The treatment material consisted of nine modules, which the patient could consult by logging on to a password-secured website. Each module involved information about mindfulness, CCRF or other related symptoms (e.g. anxiety, depression, 
anger) and audo-guided mindfulness exercises (body scans, sitting meditations, gentle yoga exercises and walking meditations). Patients were encouraged to practice and fill out their experiences in a diary on a daily basis.

\section{AAF}

AAF is a 9-week web-based physiotherapist-guided protocolled intervention [8] in which participants use an accelerometer to gain insight into their physical activity patterns. Based on their present activity level, participants received messages on their smartphone to become more or less active, in order to increase or balance their daily activities in ways that improve their energy levels. Participants described their experiences in a diary on a password-secured website and received weekly feedback from their physiotherapist.

\section{PE}

PE involved reading weekly non-reply emails, including psycho-education on fatigue, sleep hygiene, balancing energy during the day, and coping with worrying thoughts. The PE content came from the eMBCT protocol [7] and was similar in all three interventions.

\section{Measures}

\section{Outcome measure}

Fatigue severity was assessed with the 8-item CIS-FS [9]. The CIS has been validated, showed good psychometric properties and has often been used with cancer survivors [6].

\section{Moderators}

Sociodemographic and clinical moderators were assessed via self-report at T0: gender, age, education level, cancer type and fatigue duration. Baseline fatigue severity was assessed with the CISFS [10]. Fatigue catastrophizing was assessed with the Fatigue Catastrophizing Scale (FCS) [6], which showed good psychometric properties and has previously been used in cancer survivors [11]. Sense of control over fatigue (confidence about one's capacity to change fatigue) was measured with the selfefficacy scale (SE28), specifically adapted for people suffering from chronic fatigue [12]. Perceived activity and concentration were assessed with the 3 -item activity subscale and the 5 -item concentration subscale of the CIS [12]. Acceptance (non-reactive attitude to inner experiences) and presence (focus on current experiences) were assessed with the 8-item acceptance and 6-item presence subscale of the Freiburg Mindfulness Inventory (FMI) [6], which has been validated in cancer survivors, showing good 
psychometric properties [13]. The 12-item Multidimensional Scale of Perceived Social Support (MSPSS) assessed social support, which shows good psychometric properties $[13,14]$.

\section{Statistical analysis}

Analyses were based on complete cases of the treatment-adherent sample (participation in $\geq 6$ sessions). Using the SPSS macro PROCESS [15], we evaluated a set of linear regression models to examine whether the effect of treatment (eMBCT vs. AAF vs. PE) on the outcome at T2 (fatigue severity) was moderated by the moderators at T0. Following recommendations by Hayes [16], we used the T0 measure of the outcome as a covariate to correct for individual differences at baseline. The multi-categorical independent variable for treatment condition was dummy-coded, with ' 0 ' indicating absence and ' 1 ' indicating presence of each treatment condition. eMBCT served as the erence condition. Both dummy variables representing the independent variable of treatment, the moderator and the two resulting interaction terms between each dummy variable and the moderator were included in the analyses. A significant increase in explained variance $\left(\Delta R^{2}\right)$ resulting from adding the product terms to the model already containing the dummy variables and the main effect of the moderator was considered evidence for moderation [16].

Simple slope analysis were used to interpret the significant moderation effects [17]. PROCESS for SPSS provides omnibus tests, indicating if there is a difference in estimated outcome between the conditions at three different values of the moderator: 1 SD below the mean ('low'), the mean ('moderate') and 1 SD above the mean ('high'). When the omnibus test for a specific level of the moderator was significant, pairwise inferences explored if eMBCT differed from AAF or PE at that level of the moderator.

\section{Results}

\section{Study sample}

Of the 360 people who applied on the website between March 2013 and June 2015, 94 (26\%) were excluded and another 99 (28\%) declined participation, resulting in 167 participants. Of those, 132 Participants (79\%) were considered treatment-adherent (participated in $\geq 6$ sessions). Eventually, 121 participants (92\%) of the treatment-adherent sample completed the T2 assessment: eMBCT ( $n=29)$, AAF $(n=46)$ or $P E(n=46)$. Baseline characteristics of the complete cases of the treatment-adherent sample are shown in Table 1. No significant differences were found between interventions, with the exception that eMBCT participants were more severely fatigued at baseline than PE participants $(p=.021)$. 
Table 1

Baseline characteristics of treatment-adherent sample with complete data.

eMBCT

$(n=29)$

AAF $(n=46) \quad \operatorname{PE}(n=46)$

\section{Demographic characteristics, $\mathrm{n}(\%)$}

Gender

Female $\quad 18(62.1)$

$33(71.7)$

$37(80.4)$

Male

$11(37.9)$

$13(28.3)$

9 (19.6)

Age, M (SD)

$53.24(11.13)$

58.02

(9.57)

$56.15(8.66)$

Education levelab

Low/middle $10(34.5)$

$24(52.2)$

$16(34.8)$

High

$19(65.5)$

$21(45.7)$

$30(65.2)$

\section{Clinical characteristics, $\mathbf{n}(\%)$}

Type of cancer

Breast

$12(41.4)$

$18(39.1)$

$26(56.6)$

Other

$17(58.6)$

$28(60.9)$

$20(43.5)$

Length of fatigue ${ }^{a}$

$\begin{array}{llll}<2 \text { years } & 9(31.0) & 17(37.0) & 22(47.8) \\ >2 \text { years } & 20(69.0) & 28(60.9) & 24(52.2)\end{array}$

\section{Psychological characteristics, M}

(SD)

Fatigue severity (CIS-FS) ${ }^{c}$

Fatigue catastrophizing (FCS)

Sense of control over fatigue (SE28)

Perceived activity (CIS-A)

Concentration (CIS-C)

Acceptance (FMI-A)

Presence (FMI-P)

$\begin{array}{ll}44.56(6.79) \quad & 42.46 \\ & (6.60)\end{array}$

$21.99(5.36) \quad 22.05$

(5.97)

$17.52(2.42) \quad 18.15$

(2.04)

$10.38(4.83)$

10.33

(3.77)

$16.52(6.96)$

18.59

(7.21)

$20.59(3.73)$

21.63

(3.97)

$16.79(3.56)$

(3.11)

$40.15(9.35)$

$21.40(5.64)$

$18.04(2.80)$

$10.98(5.27)$

$19.24(7.56)$

$22.52(4.81)$

$18.30(3.48)$

Note. ${ }^{a}$ In AAF $n=1$ is missing; ${ }^{b}$ Low $/$ middle = primary and secondary education; High = higher vocational training and university; ${ }^{\mathrm{C}} \mathrm{eMBCT}$ participants reported a higher level of fatigue severity than PE participants $(p=.021)$. 


\begin{tabular}{|c|c|c|c|}
\hline & $\begin{array}{l}\text { eMBCT } \\
(n=29)\end{array}$ & AAF $(n=46)$ & $P E(n=46)$ \\
\hline Social support (MSPPS) & $5.31(1.18)$ & $5.75(0.94)$ & $5.68(1.24)$ \\
\hline \multicolumn{4}{|c|}{$\begin{array}{l}\text { Note. }{ }^{\mathrm{a}} \text { In AAF } \mathrm{n}=1 \text { is missing; }{ }^{\mathrm{b}} \text { Low } / \text { middle }=\text { primary and secondary education; High }=\text { higher } \\
\text { vocational training and university; }{ }^{\mathrm{c}} \text { eMBCT participants reported a higher level of fatigue severity } \\
\text { than PE participants }(p=.021) .\end{array}$} \\
\hline
\end{tabular}

\section{Moderation}

Table 2 shows the results of the linear regression models to determine moderation of treatment effect. In all analyses, there was a significant main effect of intervention on fatigue severity at $\mathrm{T} 2$, indicating that eMBCT and AAF outperformed PE in reducing fatigue severity. None of the moderation effects resulted in a significant increase in explained variance of T2 fatigue severity. Baseline fatigue severity $(p=.066)$ and fatigue catastrophizing $(p=.084)$ did show a trend towards an overall moderation effect. 
Table 2

Overall tests of moderation effects (the added value of the moderator $\mathrm{x}$ intervention interactions) on fatigue severity at T2. Moderation effects are corrected for the main effect of intervention, the main effect of the moderator and baseline fatigue severity.

\begin{tabular}{|c|c|c|c|c|c|c|}
\hline & $\begin{array}{l}\text { Full } \\
\text { model }\end{array}$ & & & $\begin{array}{l}\text { Overall test of } \\
\text { moderation effect }\end{array}$ & & \\
\hline Moderator & $F(d f)$ & $\mathrm{p}$ & $\mathrm{R}^{2}$ & $F(d f)$ & $\mathrm{p}$ & $\begin{array}{l}\mathrm{R}^{2}- \\
\text { change }\end{array}$ \\
\hline Gender (ref: males) & $\begin{array}{l}6.59(6 \\
114)\end{array}$ & .001 & .258 & $0.41(2,114)$ & .665 & .005 \\
\hline Age & $\begin{array}{l}6.56(6 \\
114)\end{array}$ & $\begin{array}{l}< \\
.001\end{array}$ & .257 & $0.31(2,114)$ & .734 & .004 \\
\hline $\begin{array}{l}\text { Education level (ref: } \\
\text { low/middle) }\end{array}$ & $\begin{array}{l}6.86(6 \\
113)\end{array}$ & .001 & .267 & $0.88(2,113)$ & .417 & .011 \\
\hline Type of cancer (ref: other) & $\begin{array}{l}7.52(6, \\
114)\end{array}$ & $<.001$ & .283 & $2.15(2,114)$ & .121 & .027 \\
\hline $\begin{array}{l}\text { Length of fatigue (ref: }<2 \\
\text { years) }\end{array}$ & $\begin{array}{l}6.74(6 \\
113)\end{array}$ & $<.001$ & .264 & $0.41(2,113)$ & .661 & .005 \\
\hline Fatigue severity at T0 & $\begin{array}{l}9.24(5, \\
115)\end{array}$ & .001 & .287 & $2.77(2,115)$ & .066 & .035 \\
\hline Fatigue catastrophizing & $\begin{array}{l}7.55(6 \\
114)\end{array}$ & $<.001$ & .285 & $2.53(2,114)$ & .084 & .032 \\
\hline $\begin{array}{l}\text { Sense of control over } \\
\text { fatigue }\end{array}$ & $\begin{array}{l}6.63(6 \\
114)\end{array}$ & $\begin{array}{l}< \\
.001\end{array}$ & .259 & $0.05(2,114)$ & .949 & $<.001$ \\
\hline Perceived activity & $\begin{array}{l}6.41(6, \\
114)\end{array}$ & $<.001$ & .252 & $0.01(2,114)$ & .988 & $<.001$ \\
\hline Concentration & $7.00(6$ & $\begin{array}{l}<. \\
.001\end{array}$ & .269 & $1.17(2,114)$ & .316 & .015 \\
\hline Acceptance & $\begin{array}{l}6.80(6 \\
114)\end{array}$ & .001 & .264 & $0.59(2,114)$ & .555 & .008 \\
\hline Presence & $\begin{array}{l}6.74(6 \\
114)\end{array}$ & .001 & .262 & $0.59(2,114)$ & .558 & .008 \\
\hline Social support & $\begin{array}{l}7.40(6, \\
114)\end{array}$ & $<.001$ & .280 & $2.17(2,114)$ & .119 & .027 \\
\hline
\end{tabular}

Regarding baseline fatigue severity (Fig. 1), the omnibus tests showed that T2 fatigue severity differed between interventions for participants with low $(F(2,115)=5.87, p=.004)$, moderate $(F(2,115)=10.53$, $p<.001)$ and high $(F(2,115)=6.70, p=.002)$ fatigue levels. Pairwise inferences showed that at low to moderate (CIS-FS=34.16 -42.09) baseline fatigue, eMBCT was outperformed by AAF $(B=-9.44(3.55)$, $\mathrm{p}=.009 ; \mathrm{B}=-4.78$ (2.09), $\mathrm{p}=.024$, respectively); no differences between eMBCT and PE. At high (CIS- 
$F S=50.01)$ baseline fatigue, eMBCT outperformed PE ( $B=8.35$ (2.73), $p=.003)$; no differences between eMBCT and AAF.

Regarding fatigue catastrophizing (Fig. 2), the omnibus tests showed that T2 fatigue severity differs between interventions at low $(F(2,114)=3.22, p=.044)$, moderate $(F(2,114)=9.88, p<.001)$ and high catastrophizing levels $(F(2,114)=9.31, p<.001)$. Pairwise inferences showed that at low catastrophizing levels ( $F C S=16.12)$, eMBCT was outperformed by $A A F(B=-6.04(2.98), p=.045)$; no differences were found between eMBCT and PE. At moderate to high ( $F C S=21.79-27.46)$ catastrophizing levels, eMBCT outperformed $P E(B=4.30$ (2.07), $p=.040 ; B=9.05$ (2.95), $p=.003$, respectively); no differences were found between $\mathrm{eMBCT}$ and AAF.

\section{Discussion}

The present study explored whether baseline sociodemographic, clinical and psychological characteristics moderated the effect of eMBCT, AAF and PE on 6-month fatigue severity. Some indication was found (marginally significant effect) that baseline fatigue severity and fatigue catastrophizing moderated the intervention effect on fatigue. None of the other characteristics moderated the intervention effect.

Regarding baseline fatigue severity, additional analyses suggested that in case of high fatigue, patients benefited more from eMBCT than from PE. These findings are in line with a recent individual patient data meta-analysis, showing that cancer patients with clinically relevant fatigue levels benefit more from psychosocial interventions than patients with non-clinical fatigue [17]. In the present study, all patients reported clinically relevant fatigue at baseline, indicating that in cases of 'extreme' fatigue eMBCT outperformed PE. Interestingly, when fatigue was less extreme but still clinically significant, AAF outperformed eMBCT, implying it seems more beneficial for patients to receive support on balancing activities in order to improve their energy levels, rather than train their awareness to help them cope with fatigue. Regarding fatigue catastrophizing, additional analyses suggested that at low catastrophizing levels, patients benefited more from AAF than from eMBCT and high catastrophizing levels, patients benefited more from eMBCT than from PE. Previous studies have shown that catastrophizing is an important predictor of fatigue severity and is linked to more daily life interference due to the fatigue [10]. Following these results, our findings suggest that when people catastrophize more, more personal support is warranted and psycho-education does not seem to offer enough support. A more intensive intervention, such as eMBCT or $A A F$, is required to help patients cope with fatigue. Similar to the moderating trend of baseline fatigue severity, patients benefit more from AAF than from eMBCT when patients catastrophize less. At these lower levels of catastrophzing, potentially less psychological factors might influence the CCRF experience and as such it can be more beneficial for patients to focus on balancing activities (AAF), rather than participate in a psychosocial intervention, such as eMBCT.

\section{Methodological Issues}


The design of this three-armed RCT in severely fatigued cancer patients provided a unique opportunity to study for which subgroup of patients what intervention is most beneficial. However, when interpreting the findings, the small sample size needs to be taken into account. The study was not powered to conduct moderation analysis and several patients did not adhere to the intervention, resulting in a small sample size. This raises the concern whether the negative findings are due to the study being underpowered rather than the effects being absent. Moreover, it might explain why we found 'only' marginally significant moderation effects of baseline fatigue severity and fatigue catastrophizing. In addition, as with other psycho-oncology research, the majority of participants were middle-aged breast cancer patients. Moreover, the sample was mainly self-selected, resulting in a group of motivated participants. Although this is mostly in line with the characteristics of cancer patients seeking psychosocial support [18], this might limit generalizability to patients with other cancer types who are less motivated to participate in an online fatigue intervention.

\section{Person-based Approach}

The present moderation study provided limited insight into 'what works best for whom?' A larger sample size could have helped us to better determine relevant moderators. However, a more person-centered approach might be more appropriate to answer this question. For example, a structured diary technique, such as the experience sampling method, in which participants receive questions multiple times a day for multiple days on end about their symptoms, thoughts and feelings, allows closely monitoring of fatigue in patients' daily living environment [19]. This results in an intensive longitudinal dataset, making it possible to examine the interactions between symptoms, cognitions, emotions and behavior in a detailed, ecologically valid manner at the level of the individual patient [20]. The network approach offers a new way to gain insight into an individual's symptom dynamics. It theorizes symptoms as elements of a complex dynamical system in which symptoms can trigger one another (e.g. sleep problems lead to fatigue and concentration problems, resulting in loss of enjoyment, which in turn can trigger a depressed mood) [20]. Such symptom networks can provide new insight into how one copes with fatigue, which could be helpful in determining what treatment would be most suitable for the individual patient [21].

\section{Conclusion}

In sum, we found some preliminary evidence that baseline fatigue severity and fatigue catastrophizing moderated the effect of eMBCT versus AAF and PE on fatigue severity in CCRF patients. These findings emphasize the potential gain in effectiveness of personalized treatment. This work could help healthcare professionals to find the right treatment for their patients suffering from CCRF. However, more research is needed to substantiate and improve guidance of personalized treatment for CCRF. The network approach [22], might help us further in answering the question 'what treatment works best for whom?'

\section{Declarations}


Funding: The work was supported by Alpe d'Huzes/Dutch Cancer society (project number 2011-5264).

Competing Interests: The authors have no relevant financial or nonfinancial interests to disclose.

Availability of data and material: Data will not be deposited. Upon request we will let the journal review the data.

Code availability: N/A.

Author Contributions: Fieke Bruggeman-Everts, Marije Wolvers, Miriam Vollenbroek-Hutten and Marije van der Lee contributed to the study conception and design. Data collection was performed by Fieke Bruggeman-Everts and Marije Wolvers. Melanie Schellekens analysed the data and wrote the first draft of the manuscript. All authors commented on previous versions of the manuscript. All authors read and approved the final manuscript.

Ethics approval: All procedures performed in studies involving human participants were in accordance with the ethical standards of the institutional and/or national research committee and with the 1964 Helsinki declaration and its later amendments or comparable ethical standards. Approval was granted by the Twente Medical Ethical Committee (P12-26).

Consent to participate: Informed consent was obtained from all individual participants included in the study.

Consent for publication: N/A.

\section{References}

1. Thong MSY, van Noorden CJF, Steindorf K, Arndt V (2020) Cancer-Related Fatigue: Causes and Current Treatment Options. Curr Treat Options Oncol 21:17. https://doi.org/10.1007/s11864-0200707-5

2. Bootsma TI, Schellekens MPJ, van Woezik RAM et al (2020) Experiencing and responding to chronic cancer-related fatigue: A meta-ethnography of qualitative research. Psychooncology 29:241-250. https://doi.org/10.1002/pon.5213

3. Mustian KM, Alfano CM, Heckler C et al (2017) Comparison of Pharmaceutical, Psychological, and Exercise Treatments for Cancer-Related Fatigue: A Meta-analysis. JAMA Oncol 3:961-968. https://doi.org/10.1001/jamaoncol.2016.6914

4. Bruggeman-Everts FZ, Wolvers MDJ, van de Schoot R et al (2017) Effectiveness of Two Web-Based Interventions for Chronic Cancer-Related Fatigue Compared to an Active Control Condition: Results of the "Fitter na kanker" Randomized Controlled Trial. J Med Internet Res 19:e336. https://doi.org/10.2196/jmir.7180

5. Wolvers MD, Bruggeman-Everts FZ, Van der Lee ML et al (2015) Effectiveness, Mediators, and Effect Predictors of Internet Interventions for Chronic Cancer-Related Fatigue: The Design and an Analysis 
Plan of a 3-Armed Randomized Controlled Trial. JMIR Res Protoc 4:e77.

https://doi.org/10.2196/resprot.4363

6. Vercoulen JHMM, Swanink CMA, Fennis JFM et al (1994) Dimensional assessment of chronic fatigue syndrome. J Psychosom Res 38:383-392. https://doi.org/10.1016/0022-3999(94)90099-X

7. Bruggeman Everts FZ, van der Lee ML, de Jager Meezenbroek E (2015) Web-based individual Mindfulness-Based Cognitive Therapy for cancer-related fatigue - A pilot study. Internet Interv 2:200213. https://doi.org/10.1016/j.invent.2015.03.004

8. Wolvers MD, Vollenbroek-Hutten MM (2015) An mHealth intervention strategy for physical activity coaching in cancer survivors. In: Proceedings of the 1st International Workshop on Personalisation and Adaptation in Technology for Health (PATH 2015). 23rd conference on User Modeling, Adaptation and Personalization. CEUR-WS, Dublin

9. Segal ZV, Williams JMG, Teasdale JD (2002) Mindfulness-Based Cognitive Therapy for depression: A new approach to preventing relapse. Guilford Press, New York, NY

10. Abrahams HJG, Gielissen MFM, Schmits IC et al (2016) Risk factors, prevalence, and course of severe fatigue after breast cancer treatment: a meta-analysis involving 12327 breast cancer survivors. Ann Oncol 27:965-974

11. Jacobsen PB, Andrykowski MA, Thors CL (2004) Relationship of catastrophizing to fatigue among women receiving treatment for breast cancer. JConsult ClinPsychol 72:355-361

12. Goedendorp MM, Gielissen MFM, Verhagen CAHHVM, Bleijenberg G (2013) Development of Fatigue in Cancer Survivors: A Prospective Follow-Up Study From Diagnosis Into the Year After Treatment. J Pain Symptom Manage 45:213-222. https://doi.org/https://doi.org/10.1016/j.jpainsymman.2012.02.009

13. Walach $\mathrm{H}$, Buchheld N, Buttenmüller V et al (2006) Measuring mindfulness-the Freiburg mindfulness inventory (FMI). Pers Individ Dif 40:1543-1555

14. Bruggeman-Everts FZ, Van der Lee ML, Van 't Hooft EFM, Nyklíček I (2017) Validation of the Dutch Freiburg Mindfulness Inventory in Patients With Medical IIIness. Sage Open 7:2158244017705936

15. Zimet GGD, Powell SS, Farley GK et al (1990) Psychometric Characteristics of the Multidimensional Scale of Perceived Social Support. J Pers Assess 55:610-617. https://doi.org/10.1207/s15327752jpa5503\&4_17

16. Hayes AF (2017) Introduction to mediation, moderation, and conditional process analysis: A regression-based approach. Guilford publications

17. Hayes AF, Montoya AK (2017) A Tutorial on Testing, Visualizing, and Probing an Interaction Involving a Multicategorical Variable in Linear Regression Analysis. Commun Methods Meas 11:1-30. https://doi.org/10.1080/19312458.2016.1271116

18. Lukkahatai N, Saligan LN (2013) Association of catastrophizing and fatigue: a systematic review. J Psychosom Res 74:100-109. https://doi.org/10.1016/j.jpsychores.2012.11.006

19. Garssen B, Van der Lee M, Van der Poll A et al (2016) Characteristics of patients in routine psychooncological care, and changes in outcome variables during and after their treatment. Psychol Heal 
31:1237-1254. https://doi.org/10.1080/08870446.2016.1204447

20. Csikszentmihalyi M, Larson R (1987) Validity and reliability of the Experience-Sampling Method. J Nerv Ment Dis 175:526-536

21. Borsboom D, Cramer AOJ (2013) Network Analysis: An Integrative Approach to the Structure of Psychopathology. Annu Rev Clin Psychol 9:91-121. https://doi.org/10.1146/annurev-clinpsy050212-185608

22. van der Lee ML, Schellekens MPJ (2020) Capturing the complexity of mental disorders in the medically ill: the network approach on behavioral medicine. Transl Behav Med 10:812-816. https://doi.org/10.1093/tbm/ibz019

\section{Figures}

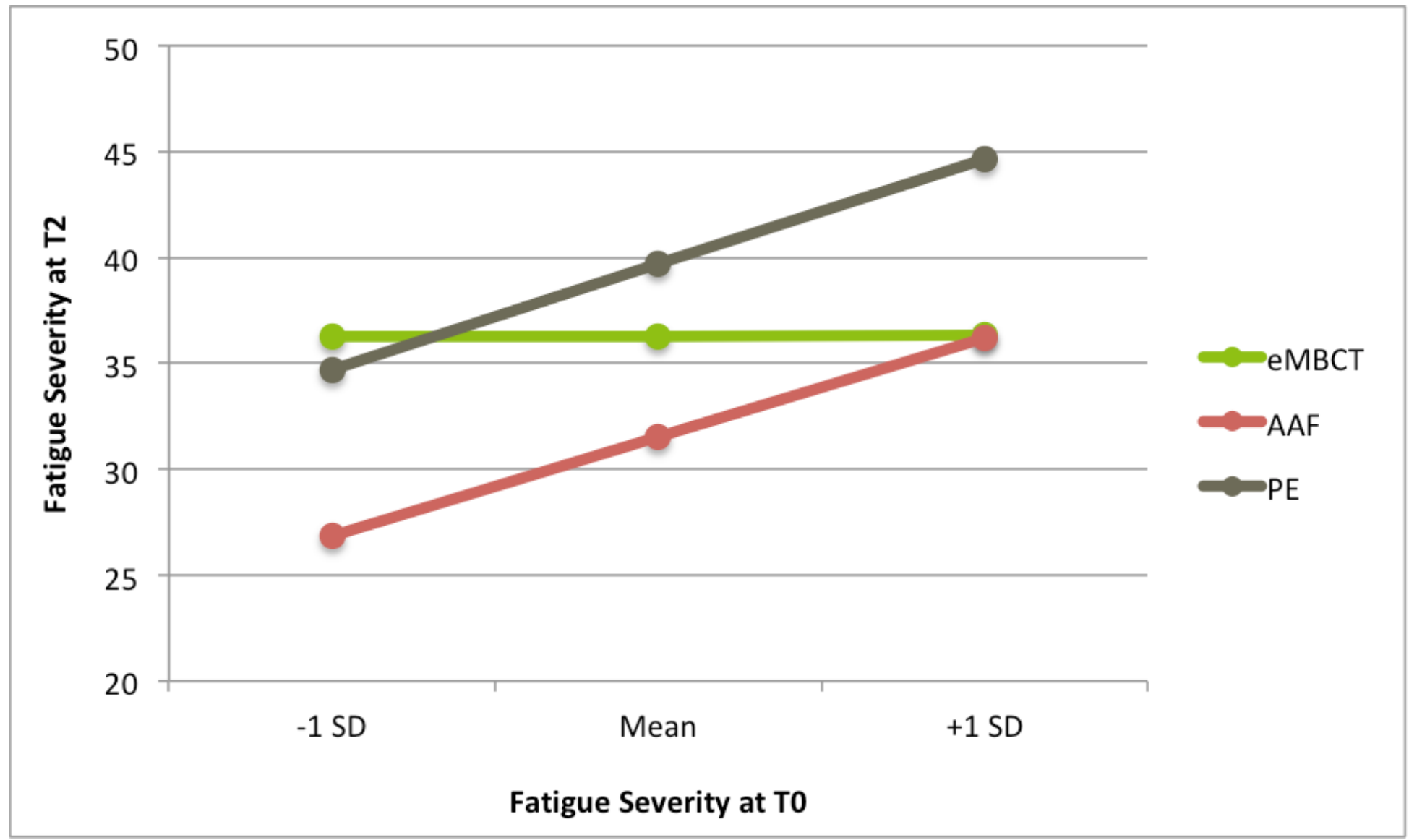

Figure 1

The effect of treatment on T2 fatigue severity at different levels (-1 SD, Mean, +1 SD) of the moderator baseline fatigue severity. 


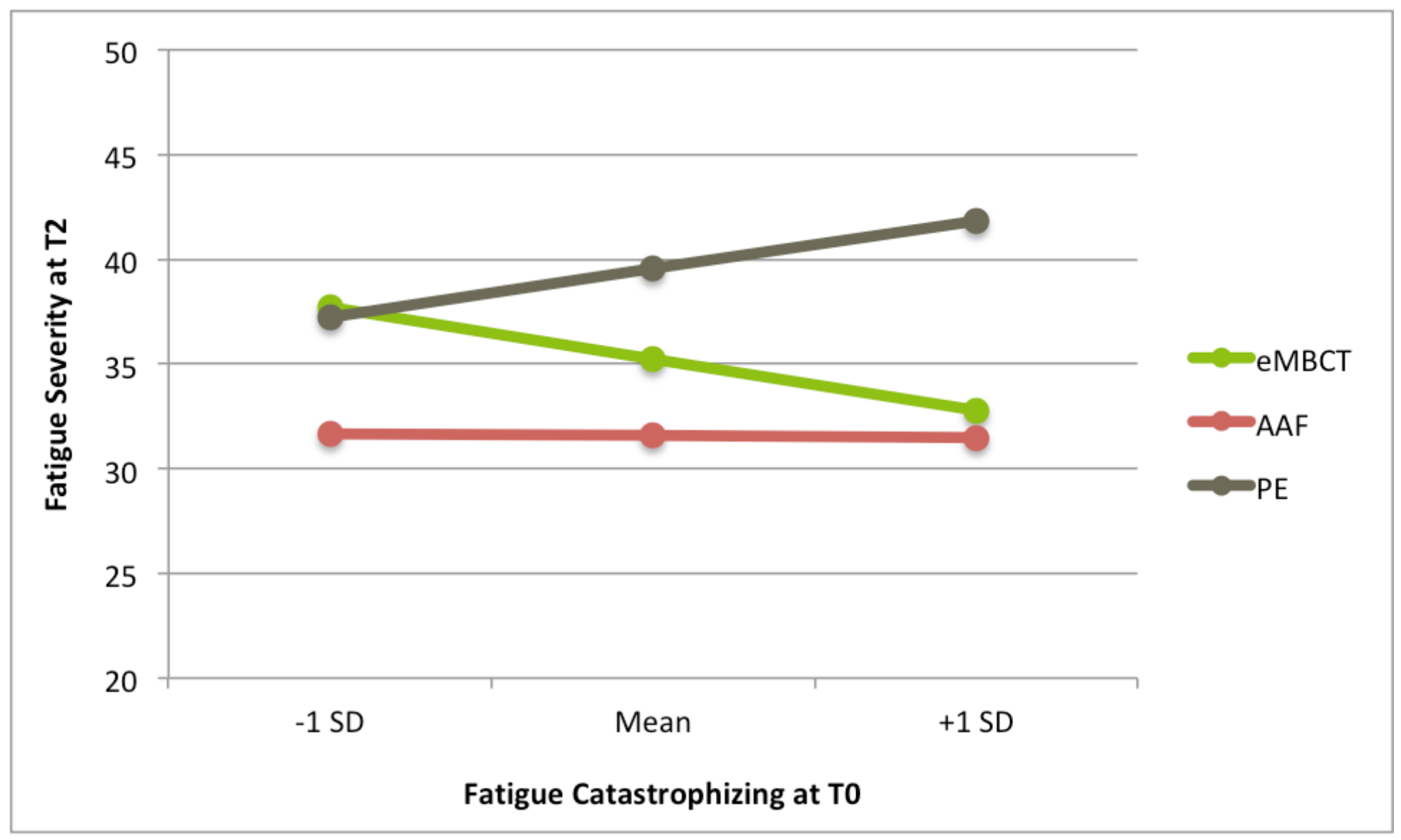

Figure 2

The effect of treatment on T2 fatigue severity at different levels (-1 SD, Mean, +1 SD) of the moderator fatigue catastrophizing. 